\title{
CONJECTURES SUR L'ÉTAT SMECTIQUE
}

\author{
P. G. de GENNES
}

Faculté des Sciences, Orsay, et C. E. N., Saclay

\begin{abstract}
Résumé. - On présente une description phénoménologique des déformations dans un smectique impliquant deux paramètres scalaires : le déplacement $u$ des couches perpendiculairement à leur plan et la dilatation en volume $\theta$. L'intensité de la lumière diffusée par les fluctuations de $u$ et de $\theta$ est faible sauf lorsque le vecteur de diffusion est presque parallèle au plan des couches. On analyse aussi le spectre des vibrations de grande longueur d'onde : pour un vecteur d'onde q oblique par rapport au plan des couches, on attend deux branches acoustiques observables en effet Brillouin.
\end{abstract}

Abstract. - A continuum theory for smectic liquid crystals is constructed in terms of two scalar functions : the displacement $u$ of the layers normal to their plane and the volume dilatation $\theta$. The intensity of the light scattering due to thermal fluctuations of $u$ and of $\theta$ is expected to be weak - the only exception corresponding to a scattering wave vector parallel to the plane of the layers. The frequency spectrum of the vibrations (for a wave-vector $q$ neither parallel nor normal to the optical axis) shows two acoustic branches which should be observable by Brillouin scattering.

I. Introduction et description de Landau-Peierls. - Les smectiques sont des matériaux stratifiés, avec, dans la configuration au repos, des couches successives équidistantes (intervalle $d$ ) et perpendiculaires à une même direction $\mathrm{OZ}$ (Fig. 1). La structure détaillée

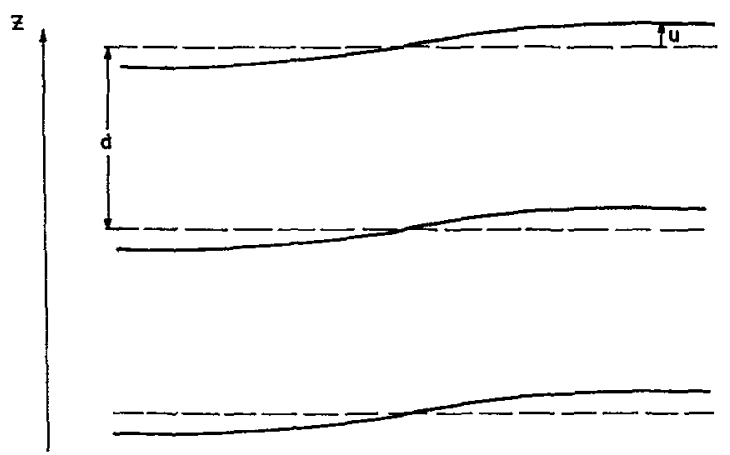

FIG. 1. - Représentation schématique de l'état smectique. Lignes pointillées : plan des couches dans la configuration au repos. Lignes pleines : aspect de la déformation dans le mode d'ondulation $\left(q_{z}=0\right)$.

de ces couches est en général mal connue [1]. Nous nous contentons ici d'admettre que de tels systèmes existent, avec les symétries suivantes :
1) pas d'ordre à longue distance dans le plan de chaque couche (différence avec un cristal tridimensionnel),

2) aucun axe privilégié dans le plan de la couche (système optiquement uniaxe d'axe $z$ ),

3) les directions $+\mathrm{Oz}$ et $-\mathrm{Oz}$ sont équivalentes (smectique non ferroélectrique).

Partant d'une telle configuration au repos, nous voulons faire ici une analyse phénoménologique des déformations de grande longueur d'onde. Ceci a déjà été entrepris dans le passé, en partant de deux points de vue un peu différents :

a) Le point de vue d'Oseen [2], remanié par Frank [3], peut être présenté de la façon suivante : repérons en chaque point $r$ l'axe optique local par un vecteur unitaire $\mathbf{n}(\mathbf{r})$ ou « directeur ». La forme la plus générale pour l'énergie libre de déformation est alors (par $\mathrm{cm}^{3}$ )

$$
\begin{aligned}
F=F_{0}+1 / 2 K_{1}(\operatorname{div} \mathbf{n})^{2}+ & 1 / 2 K_{2}\left(\mathbf{n} \cdot \operatorname{rot} \mathbf{n}+q_{0}\right)^{2}+ \\
& +1 / 2 K_{3}\left(\mathbf{n}_{\wedge} \operatorname{rot} \mathbf{n}\right)^{2} .
\end{aligned}
$$

Telle quelle, cette forme s'applique aux cholestériques $\left(q_{0} \neq 0\right)$ ou aux nématiques $\left(q_{0}=0\right)$, qui sont aussi des matériaux localement uniaxes, mais sans structure en couches. Dans les smectiques, il y a une exigence 
supplémentaire : pour un système de couches incompressibles, l'intégrale

$$
\frac{1}{d} \int_{\mathbf{A}}^{B} \mathbf{n} \cdot \mathrm{d} \mathbf{r}
$$

représente le nombre de couches traversées le long d'un chemin reliant $A$ à $B$. Ce nombre doit être indépendant du chemin choisi, donc

$$
\operatorname{rot} \mathbf{n} \equiv \mathbf{0} \text {. }
$$

On est alors amené à une énergie libre qui contient uniquement la divergence de $\mathbf{n}$

$$
F=F_{0}+1 / 2 K_{1}(\operatorname{div} \mathbf{n})^{2}-1 / 2 \chi_{\mathrm{a}}(\mathbf{n} \cdot \mathbf{H})^{2} .
$$

On a ajouté dans cette dernière expression l'effet d'un champ magnétique $\mathbf{H} \cdot \chi_{\mathrm{a}}=\chi_{\|}-\chi_{\perp}$ est l'anisotropie de la susceptibilité magnétique (on se limitera ici au cas $\chi_{a}>0$ ).

b) La description de Peierls et Landau [4] adopte comme variable fondamentale le déplacement unidimensionnel $u(\mathbf{r})$ des couches perpendiculairement à leur plan. Pour de faibles déplacements, l'énergie libre a alors la forme

$$
\begin{aligned}
& F=F_{0}+\frac{\bar{B}}{2}\left(\frac{\partial u}{\partial z}\right)^{2}+\frac{\chi_{\mathrm{a}} H^{2}}{2}\left[\left(\frac{\partial u}{\partial x}\right)^{2}+\left(\frac{\partial u}{\partial y}\right)^{2}\right] \\
& +\frac{1}{2} K_{1}\left(\frac{\partial^{2} u}{\partial x^{2}}+\frac{\partial^{2} u}{\partial y^{2}}\right)^{2}+\frac{1}{2} K^{\prime}\left(\frac{\partial^{2} u}{\partial z^{2}}\right)^{2}+ \\
& +K^{\prime \prime} \frac{\partial^{2} u}{\partial z^{2}}\left(\frac{\partial^{2} u}{\partial x^{2}}+\frac{\partial^{2} u}{\partial y^{2}}\right) \text {. }
\end{aligned}
$$

Le champ $H$ est ici supposé parallèle à la direction de l'axe optique au repos $\mathrm{Oz}$. Pour $H=0$ il n'y a pas de terme en $\left(\frac{\partial u}{\partial x}\right)^{2}$ par exemple, car un tel terme impliquerait une valeur de $F-F_{0}$ non nulle pour une rotation d'ensemble du système par rapport à $\mathrm{Oy}$. L'expression (I.5) ne contient pas le terme en

$$
\frac{\partial u}{\partial z}\left(\frac{\partial^{2} u}{\partial x^{\prime}}+\frac{\partial^{2} u}{\partial y^{\prime}}\right)
$$

de la réf. [4] : ce terme disparaît en effet lorsque les directions $+\mathrm{Oz}$ et $-\mathrm{Oz}$ sont équivalentes. En pratique les termes $K^{\prime}$ et $K^{\prime \prime}$ sont négligeables par rapport au terme $B$, et peuvent être omis. Par contre le terme $K_{1}$ doit être conservé, car il figure seul (en champ $H$ nul) lorsque les déformations sont indépendantes de la coordonnée $z\left(\frac{\partial u}{\partial z}=0\right)$.
Il est facile de vérifier que les définitions (I.4) et (I.5) du coefficient $K_{1}$ coïncident.

Au total, la description de Peierls-Landau recouvre celle de Oseen (pour les déformations faibles) mais tient compte en plus (par le terme $\bar{B}$ ) d'une modification éventuelle de la distance entre couches. On peut exploiter la formule (I.5) en passant aux composantes de Fourier :

$$
u_{q}=\int \mathrm{d} \mathbf{r} u(\mathbf{r}) \mathrm{e}^{i q \cdot \mathbf{r}}
$$

$$
\begin{aligned}
F-F_{0} & = \\
& =\frac{1}{2} \sum_{q}\left|u_{q}\right|^{2}\left\{\bar{B} q_{z}^{2}+K_{1}\left(q_{\perp}^{2}+\xi^{-2}\right) q_{\perp}^{2}\right\}
\end{aligned}
$$

où l'on a posé $q_{1}^{2}=q_{x}^{2}+q_{y}^{2}$ et où l'on a introduit la longueur de cohérence magnétique $\xi$, définie par [5]

$$
\xi=\sqrt{\frac{K_{1}}{\chi_{\mathrm{a}}}} \frac{1}{H} .
$$

En appliquant le théorème d'équipartition à (I.6) on obtient finalement les moyennes thermodynamiques

$$
\begin{aligned}
& <\left|u_{q}\right|^{2}>=\frac{k_{B} T}{\bar{B} q_{z}^{2}+K_{1}\left(q_{\perp}^{2}+\xi^{-2}\right) q_{\perp}^{2}} \\
& <u^{2}(\mathbf{r})>=\frac{k_{B} T}{4 \pi\left(\bar{B} K_{1}\right)^{1 / 2}} \log (\xi / d) .
\end{aligned}
$$

Pour $H \rightarrow 0,<u^{2}>$ a une divergence logarithmique, qui est discutée dans la réf. [4]. A cause de cette divergence, la notion d'ordre smectique n'est pas rigoureusement self consistente en champ nul. Nous avons calculé ailleurs les corrélations qui subsistent alors, sur un exemple soluble à deux dimensions [6]. Mais, pour les systèmes qui nous intéressent ici, il importe de réaliser que la divergence logarithmique est très faible : considérons par exemple un grand monocristal smectique ayant atteint son équilibre dans le champ terrestre. Avec $E \sim 10^{11} \mathrm{ergs} / \mathrm{cm}^{3}$ $K_{1} \sim 10^{-6}$ dyne, $\chi_{\mathrm{a}} \sim 10^{-7}$ on, attend $\sqrt{\left\langle u^{2}>\right.} \sim 1 \AA$. Donc en pratique $\left\langle u^{2}\right\rangle$ n'est pas beaucoup plus grand que dans un solide (soit à cause d'un champ résiduel, soit en raison des dimensions finies du spécimen).

Si nous revenons maintenant sur le principe général de la description de Landau-Peierls, nous constatons que son hypothèse fondamentale est d'utiliser comme seule variable d'état le déplacement unidimensionnel des couches $u(\mathbf{r})$. Or, en réalité, pour spécifier à l'échelle macroscopique l'état d'un petit élément smectique, il faut non seulement connaître la configuration des 
couches, décrite par $u(\mathbf{r})$, mais aussi la densité $v$ d'atomes présente dans chaque couche. On peut par exemple définir une dilatation transversale $\psi$ en posant

$$
v=v_{0}(1-\psi)
$$

où $v_{0}$ est la valeur au repos. La dilatation en volume $\theta$ est reliée à $\psi$ et $u$ par la formule

$$
\theta=\psi+\frac{\partial u}{\partial z}
$$

Dans ce qui suit, nous décrirons l'état local de déformation du smectique par les variables $u(\mathbf{r})$ et $\theta(\mathbf{r})$. Nous étudierons d'abord les propriétés statiques (énergie libre et intensité de la lumière diffusée par les fluctuations thermiques) puis les propriétés dynamiques (énergie et spectres de vibration).

Nous vérifierons que l'introduction de la variable $\theta$ était effectivement nécessaire, et ce pour deux raisons :

a) les fluctuations thermiques de $u$ et $\theta$ donnent des contributions comparables en diffusion de la lumière,

b) les mouvements de $u$ et de $\theta$ s'effectuent à des fréquences comparables et sont fortement couplés.

Ces propriétés ( $a$ ) et $(b)$ sont radicalement opposées à ce que l'on rencontre dans les nématiques, où $(a)$ la diffusion de la lumière est dominée par les fluctuations d'orientation (les fluctuations de $\theta$ ne représentant qu'une faible correction [5]), et (b) les relaxations d'orientation sont lentes par rapport à celles de $\theta$ [7].

II. Statique des fluctuations - En l'absence d'effets piézoélectriques, avec les deux variables d'état $u(\mathbf{r})$ et $\theta(\mathbf{r})$ la forme de l'énergie libre de déformation (par $\mathrm{cm}^{3}$ ) est :

$$
\begin{aligned}
F=\frac{1}{2} A \theta^{2} & +\frac{1}{2} B \gamma^{2}+C \theta \gamma \\
& +\frac{1}{2} \chi_{\mathrm{a}} H^{2}\left[\left(\frac{\partial u}{\partial x}\right)^{2}+\left(\frac{\partial u}{\partial y}\right)^{2}\right] \\
& +\frac{1}{2} K_{1}\left(\frac{\partial^{2} u}{\partial x^{2}}+\frac{\partial^{2} u}{\partial y^{2}}\right)^{2}
\end{aligned}
$$

où $\gamma \equiv \frac{\partial u}{\partial z}$, et les coefficients $A, B, C$ représentent des rigidités isothermes (la stabilité exige $A B>C^{2}$ ). Pour une configuration statique de $u$ imposée la valeur d'équilibre de la dilatation $\theta$ est

$$
\theta=-\frac{C}{A} \frac{\partial u}{\partial z}
$$

En réinsérant cette valeur dans (II.1), on retrouve la forme de Landau-Peierls avec :

$$
\vec{B}=B-\frac{C^{2}}{A} \quad(>0)
$$

Mais la relation (II.2) n'est pas applicable aux fluctuations thermiques qui nous intéressent en vue de la diffusion de la lumière. Nous conservons donc la forme (II.1), qui, après transformation de Fourier, devient :

$$
\begin{aligned}
F & =\sum_{q} F_{q} \\
F_{q} & =\frac{1}{2} A\left|\theta_{q}\right|^{2}+\frac{1}{2} B^{\prime}\left|\gamma_{q}\right|^{2}+C \theta_{q} \gamma_{-q} \\
B^{\prime} & =B+K_{1} \frac{q_{\perp}^{2}\left(q_{\perp}^{2}+\xi^{-2}\right)}{q_{z}^{2}}
\end{aligned}
$$

On en tire les moyennes thermodynamiques:

$$
\left.\begin{array}{l}
<\left|\theta_{q}\right|^{2}>=\frac{\int\left|\theta_{q}\right|^{2} \mathrm{e}^{-\beta F_{q}} \mathrm{~d} \theta_{q}}{\int \mathrm{e}^{-\beta F_{q}} \mathrm{~d} \theta_{q}} \text { etc. } \\
<\left|\theta_{q}\right|^{2}>=\frac{B^{\prime} k_{B} T}{A B^{\prime}-C^{2}} \\
<\left|\gamma_{q}\right|^{2}>=\frac{A k_{B} T}{A B^{\prime}-C^{2}} \\
<\theta_{q} \gamma_{-q}>=\frac{-C k_{B} T}{A B^{\prime}-C^{2}}
\end{array}\right\}
$$

Examinons maintenant l'effet de ces fluctuations sur la diffusion de la lumière. Dans la configuration au repos, le tenseur constante diélectrique a pour éléments non nuls $\varepsilon_{z z}=\varepsilon_{\|}$et $\varepsilon_{x x}=\varepsilon_{y y}=\varepsilon_{\perp}\left(\varepsilon_{\|}\right.$et $\varepsilon_{\perp}$ sont les valeurs relatives à la fréquence optique considérée). Au premier ordre en déformations, les modifications $\mathrm{du}$ tenseur diélectrique ont la forme :

$$
\begin{aligned}
& \delta \varepsilon_{z z}=a_{z} \theta+b_{z} \gamma \\
& \delta \varepsilon_{x x}=\delta \varepsilon_{y y}=a_{\perp} \theta+b_{\perp} \gamma \\
& \delta \varepsilon_{x y}=0 \\
& \delta \varepsilon_{z x}=\left(\varepsilon_{\perp}-\varepsilon_{\|}\right) \frac{\partial u}{\partial x} \\
& \delta \varepsilon_{z y}=\left(\varepsilon_{\perp}-\varepsilon_{\|}\right) \frac{\partial u}{\partial y} .
\end{aligned}
$$


Dans ces formules, $a_{z}, a_{\perp}, b_{z}, b_{\perp}$ sont quatre coefficients phénoménologiques sans dimensions, et de l'ordre de l'unité. La forme simple pour $\delta \varepsilon_{z x}$ (ou $\delta \varepsilon_{z y}$ ) vient de ce que $u / x=$ constante correspond à une rotation pure (au $1^{\mathrm{e}}$ ordre en $u$ ) autour de Oy.

Lorsque la différence $\left|\varepsilon_{\perp}-\varepsilon_{\|}\right|$est faible, l'intensité de la lumière diffusée, pour un vecteur de diffusion $\mathbf{q}$, est proportionnelle à [8]

$$
I(\mathbf{q})=\left\langle|\mathbf{i} . \delta \hat{\varepsilon}(\mathbf{q}) \cdot \mathbf{f}|^{2}\right\rangle
$$

où $\mathbf{i}$ et $\mathbf{f}$ sont des vecteurs unitaires repérant les polarisations initiale et finale, et $\delta \widehat{\varepsilon(\mathbf{q})}$ la transformée de Fourier du tenseur (II.6). On peut calculer $I(q)$ à partir de (II.5) et (II.6).

Les conclusions générales sont les suivantes :

a) si $\mathbf{i}$ et $\mathbf{j}$ sont parallèles entre eux, et de surcroît soit parallèles, soit perpendiculaires à l'axe optique $\mathrm{Oz}$, l'intensité diffusée est faible. Par exemple :

$$
\begin{aligned}
& <\left|\delta \varepsilon_{z z}(\mathbf{q})\right|^{2}>= \\
& \quad=\frac{k_{B} T}{A B^{\prime}-C^{2}}\left\{a_{z}^{2} B^{\prime}-2 a_{z} b_{z} C+b_{z}^{2} A\right\}
\end{aligned}
$$

En ordre de grandeur

$$
<\left|\delta \varepsilon_{z z}(\boldsymbol{q})\right|^{2}>\sim \frac{k_{B} T}{A}
$$

comme pour un fluide isotrope de compressibilité $A^{-1}$.

b) Le cas le plus intéressant correspond à une polarisation incidente i parallèle à l'axe optique, et une polarisation sortante $\mathbf{f}$ perpendiculaire à cet axe (ou l'inverse).

Par exemple, pour $\mathbf{f}$ parallèle à $\mathrm{Ox}$, on mesure

$$
<\left|\delta \varepsilon_{z x}(\mathbf{q})\right|^{2}>=\frac{\left(\varepsilon_{\perp}-\varepsilon_{\|}\right)^{2} k_{B} T A q_{x}^{2}}{B^{\prime} A-C^{2}} .
$$

En général, pour $q_{z} \neq 0$, cette intensité est faible elle aussi. Mais pour $q_{z}=0$ (vecteur de diffusion normal à l'axe optique) elle devient grande. Par exemple, avec $q$ parallèle à $O x$, on obtient :

$$
<\left|\delta \varepsilon_{z x}(q)\right|^{2}>=\frac{\left(\varepsilon_{\perp}-\varepsilon_{\|}\right)^{2} k_{B} T}{K_{1}\left(q^{2}+\xi^{-2}\right)} .
$$

Cette expression est très analogue, en forme et en ordre de grandeur, à ce que l'on a dans un nématique [9] [8]. Mais la forte diffusion décrite par (II.10) est restreinte à un très petit domaine en $\mathbf{q}$. Plus précisément il faut réaliser la condition

$$
B q_{z}^{2}<K_{1} q^{4}
$$

soit pour l'angle $\Phi$ entre $\mathbf{q}$ et le plan $\mathrm{xOy}$ :

$$
\Phi \sim \frac{q_{z}}{q}<\sqrt{\frac{K_{1}}{B}} q
$$

$\sqrt{\frac{K_{1}}{B}}$ sera de l'ordre de la distance intermoléculaire $d$ et cette condition peut être écrite en gros

$$
\Phi<q d\left(\sim 10^{-2}\right)
$$

c'est-à-dire que l'angle entre $q$ et le plan xOy doit être inférieur à $\sim 1 / 2$ degré. Il n'est pas sûr que cette condition puisse être réalisée sur l'ensemble du spécimen, au moins pour les cristaux liquides smectiques assez imparfaits dont on dispose couramment. Mais la recherche de ce pic de diffusion pour $\Phi=0$ mériterait en tout cas d'être tentée.

III. Dynamique. - Dans un solide nous trouvons pour chaque vecteur d'onde $\mathbf{q}$ trois modes de propagation acoustique (Fig. 2a). Dans un liquide isotrope nous avons un mode acoustique (oscillations de densité) et deux modes transverses complètement amortis, contrôlés par la viscosité Im $\omega=\eta q^{2} / \rho$ (où $\eta$ est la viscosité, $\rho$ la densité) (Fig. 2b). Qu'advient-il dans un smectique ?

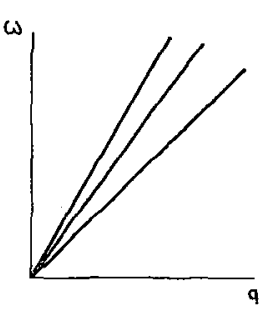

(a)

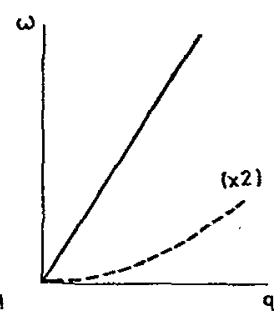

(b)

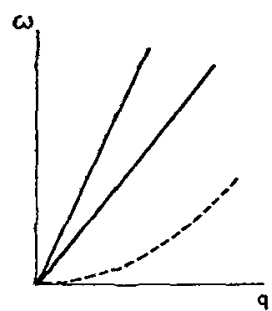

(c)
FIG. 2. - Spectre des excitations basse fréquence pour un solide (a), un liquide isotrope (b), et un smectique (c). Les branches en trait plein représentent des fréquences réelles, les branches pointillées des fréquences imaginaires (régions purement amorties).

Comme nous allons le voir, il y a deux types de comportement possibles selon l'orientation de $q$ par rapport à l'axe optique :

a) pour $q_{z} \neq 0$ on trouve deux branches acoustiques (vibrations couplées de $\theta$ et de $\gamma=\partial u / \partial z$ ) plus une branche amortie transversale analogue à celle d'un liquide (Fig. $2 c$ ). 
b) pour $q_{z}=0$ on attend un mode de compression acoustique (vibrations de $\theta$ ), un mode d'ondulation (Fig. 1) fortement amorti, et un mode hydrodynamique (glissement plan sur plan avec frottement visqueux).

Etudions d'abord le cas $q_{z} \neq 0$. Ici nous allons avoir des vibrations acoustiques de relativement haute fréquence, pour lesquelles le comportement est 1) adiabatique, 2) peu amorti. Pour engendrer ces vibrations il faut partir de l'énergie (et non plus de l'énergie libre) qui a la forme $\left(\right.$ par $\mathrm{cm}^{3}$ )

$$
E=E_{0}+1 / 2 A_{0} \theta^{2}+1 / 2 B_{0} \gamma^{2}+C_{0} \theta \gamma
$$

Dans cette expression $A_{0}, B_{0}, C_{0}$ sont des coefficients de rigidité adiabatique. Les termes impliquant des dérivées d'ordre supérieur (comme le terme en $K_{1}$ de (II.1)) et les termes associés au champ magnétique ne sont pas importants pour $q_{z} \neq 0$ et ont été omis dans (III.1). Le système de tensions mécaniques $\sigma_{\alpha \beta}$ résultant de (III.l) peut être calculé par le travail fourni à la surface d'un spécimen déformé, et il se réduit aux composantes suivantes :

$$
\left.\begin{array}{l}
\sigma_{z z}=\left(B_{0}+C_{0}\right) \gamma+\left(A_{0}+C_{0}\right) \theta \\
\sigma_{x x}=\sigma_{y y}=C_{0} \gamma+A_{0} \theta
\end{array}\right\}
$$

Le champ de vitesses dans le smectique est un vecteur de composantes $\left(v_{x}, v_{y}, \partial u / \partial t\right)$. En négligeant l'amortissement, les équations d'accélération sont :

$$
\begin{aligned}
\rho \frac{\partial^{2} u}{\partial t^{2}}=\frac{\partial}{\partial z} & \sigma_{z z}= \\
& =\left(B_{0}+C_{0}\right) \frac{\partial \gamma}{\partial z}+\left(A_{0}+C_{0}\right) \frac{\partial \theta}{\partial z}
\end{aligned}
$$

$\rho \frac{\partial v_{x}}{\partial t}=\frac{\partial}{\partial x} \sigma_{x x}=C_{0} \frac{\partial \gamma}{\partial x}+A_{0} \frac{\partial \theta}{\partial x}$

$\rho \frac{\partial v_{y}}{\partial t}=C_{0} \frac{\partial \gamma}{\partial y}+A_{0} \frac{\partial \theta}{\partial y}$

Il faut y adjoindre l'équation de conservation

$$
\frac{\partial v_{x}}{\partial x}+\frac{\partial v_{y}}{\partial y}+\frac{\partial}{\partial t}(y-\theta)=0
$$

En cherchant des modes propres de la forme

$$
\exp \{i \mathbf{q} \cdot \mathbf{r}-i \omega t\}
$$

on aboutit à l'équation séculaire

$$
\begin{gathered}
\omega\left\{\left[\rho \omega^{2}-\left(B_{0}+C_{0}\right) q_{z}^{2}\right]\left[\rho \omega^{2}-A_{0} q_{\perp}^{2}\right]-\right. \\
\left.-\left(A_{0}+C_{0}\right) q_{z}^{2}\left[\rho \omega^{2}+C_{0} q_{\perp}^{2}\right]\right\}=0 .
\end{gathered}
$$

Le mode $\omega=0$ correspond à des vitesses hydrodynamiques perpendiculaires à $\mathbf{q}$ et à l'axe optique $\mathrm{Oz}$. (Dans une approximation plus raffinée, tenant compte de la friction, il prendrait la forme

$$
\rho \omega=i\left(q_{z}^{2} \eta_{z}+q_{\perp}^{2} \eta_{\perp}\right)
$$

où $\eta_{z}$ et $\eta \perp$ sont deux coefficients de viscosité.)

Les deux autres racines de (III.6) sont les plus intéressantes, car elles décrivent les modes acoustiques qui sont seuls couplés en pratique à la diffusion de la lumière. On peut les mettre sous la forme :

$$
\begin{aligned}
& \omega_{1}=s_{1}(\Phi) q \\
& \omega_{2}=s_{2}(\Phi) q
\end{aligned}
$$

où $\Phi$ est l'angle entre $\mathrm{Oz}$ et $x$. En posant $\rho s^{2}=\mathbf{q}$, on obtient $\rho s_{1}^{2}$ et $\rho s_{2}^{1}$ comme les racines (positives) de l'équation :

$$
\begin{aligned}
x^{2}-x & \left\{A_{0} \cos ^{2} \Phi+\left(A_{0}+B_{0}+2 C_{0}\right) \sin ^{2} \Phi\right\}+ \\
& +\sin ^{2} \Phi \cos ^{2} \Phi\left\{B_{0} A_{0}-C_{0}^{2}\right\}=0 . \text { (III. } 7
\end{aligned}
$$

En général les vitesses $s_{1}$ et $s_{2}$ seront comparables à la vitesse du son dans la phase liquide isotrope. Il faut noter toutefois que l'une des vitesses tend vers 0 quand $\Phi \rightarrow \pi / 2$ (q paralèlle à l'axe optique) et aussi quand $\Phi \rightarrow 0$ (q perpendiculaire à l'axe optique). Nous allons maintenant discuter brièvement le cas $\Phi=0$, qui est particulièrement intéressant puisqu'il correspond aux fortes diffusions de la lumière.

Pour $q_{z}=0$ nous avons un mode hydrodynamique banal $\left(\rho \omega=i \eta q^{2}\right)$ et un mode acoustique de vitesse $\sqrt{A_{0}} / \rho$, qui tous deux contribuent peu à la diffusion. Le mode véritablement intéressant est le mode d'ondulation (Fig. 1). Dans la discussion de ce mode, il faut inclure le terme $K_{1}$ de l'équation (II.1) qui décrit la seule force de rappel présente. Il faut aussi tenir compte des amortissements, qui font intervenir jci un troisième coefficient de viscosité $\eta_{u}$. Au total, on obtient alors pour $u$ l'équation de mouvement :

$$
-\rho \omega^{2} u=-K_{1} q^{2}\left(q^{2}+\xi^{-2}\right) u-i \omega \eta_{u} q^{2} u
$$

Considérons d'abord le cas sans champ magnétique $(\xi=\omega)$ et limitons-nous plus particulièrement au domaine

$$
\frac{K_{1} \rho}{\eta_{u}^{2}} \ll 1
$$


Dans ces conditions on trouve à partir de (III.8) un mode de relaxation purement hydrodynamique

$$
\omega_{1}=+i \eta_{u} q^{2}
$$

et une racine décrivant les fluctuations d'orientation :

$$
\omega_{2}=+i \frac{K_{1} q^{2}}{\eta_{u}} .
$$

C'est cette dernière racine qui définit l'élargissement de la raie Rayleigh observable pour $q_{z}=0$. Sa dépendance en $q$ est très analogue à ce que l'on attend dans un nématique [7].

Terminons cette discussion du mode d'ondulation par une remarque concernant le cas de champs magnétiques $H$ très forts : plus précisément, supposons que les inégalités suivantes sont réalisées :

$$
\left.\begin{array}{l}
q \xi<1 \\
q \eta_{u}<H \sqrt{\chi_{\mathrm{a}} \rho}
\end{array}\right\}
$$

Alors les termes réactifs dominent sur les termes dissipatifs, et on attend d'après (III.8) une onde " magnétoacoustique » lente, de vitesse

$$
S_{H}=H \sqrt{\frac{\chi_{\mathrm{a}}}{\rho}} \sim \mathrm{qq} \mathrm{cm} / \mathrm{s} .
$$

Mais la deuxième inégalité (III.10) implique des valeurs de $q$ extrêmement faibles qui seraient très difficiles à réaliser en pratique.

IV. Remarques finales. - Notre description phénoménologique des smectiques prévoit :

a) une diffusion lumineuse remarquable lorsque le vecteur de diffusion $\mathbf{q}$ est perpendiculaire à l'axe optique. Dans ce cas, et si en outrel'inégalité $K_{1} \rho \ll \eta_{u}^{2}$ est satisfaite, le spectre en fréquence de la lumière diffusée devrait correspondre à un pic central étroit, dont la largeur est définie par l'équation (III.9),

b) dans les autres directions de $\mathbf{q}$, une diffusion Brillouin d'intensité comparable à ce qu'elle est dans un fluide isotrope, mais comportant deux branches acoustiques.

Il faut insister ici sur certaines limitations de notre traitement :

1) La divergence logarithmique des fluctuations de position des couches (en champ magnétique nul) a été ignorée complètement. Comme nous l'avons vu dans l'introduction, cette divergence est faible, et probablement observable non pas dans le domaine visible $(q d \ll 1)$ mais seulement dans le domaine des rayons $X(q d \sim 1)$.

2) Nous nous sommes limités à deux variables d'état, le déplacement des couches $u$ et la dilatation en volume $\theta$. On peut envisager aussi d'inclure la température $T$, mais les effets qui en résultent (diffusion de la lumière par les fluctuations de $T$, amortissement par conduction thermique des vibrations) sont en général faibles et peu intéressants. Dans un cas toutefois les effets thermiques sont peut-être non triviaux : celui du mode d'ondulation $\left(q_{z}=0\right)$; il faudrait une étude plus détaillée pour savoir si ce mode est adiabatique ou isotherme [10].

3) Une autre variable éventuellement importante est le champ électrique $\mathbf{E}$, puisqu'une déformation de la structure smectique fait en général apparaître des polarisations et des charges [11]. Il est possible de montrer toutefois que dans un smectique la forme de Landau-Peierls pour l'énergie libre (éq. I.5) n'est pas sérieusement affectée par ces effets. L'argument est le suivant : la polarisation $\mathbf{P}$ associée aux déplacements $u$ a la forme :

$$
\begin{aligned}
& P_{z}=-\mathrm{e}_{1}\left(\frac{\partial^{2} u}{\partial x^{2}}+\frac{\partial^{2} u}{\partial y^{2}}\right)-\mathrm{e}_{2} \frac{\partial^{2} u}{\partial z^{2}} \\
& P_{x}-\mathrm{e}_{3} \frac{\partial^{2} u}{\partial z \partial x} \\
& P_{y}=-\mathrm{e}_{3} \frac{\partial^{2} u}{\partial z \partial y}
\end{aligned}
$$

La densité de charges correspondante est :

$$
-\operatorname{div} \mathbf{P}=\left(\mathrm{e}_{1}+\mathrm{e}_{3}\right)\left(\frac{\partial^{2} \gamma}{\partial x^{2}}+\frac{\partial^{2} \gamma}{\partial y^{2}}\right)+\mathrm{e}_{2} \frac{\partial^{2} \gamma}{\partial z^{2}}
$$

L'équation de Poisson pour le potentiel électrique $V$ est:

$$
\operatorname{div}(\hat{\varepsilon} \nabla V)=4 \pi \operatorname{div} \mathbf{P}
$$

(où $\varepsilon$ est ici le tenseur constante diélectrique à fréquence nulle).

Soit pour une composante de Fourier de vecteur d'onde $\mathbf{q}$ :

$$
\begin{aligned}
-\left(\varepsilon_{\|} q_{z}^{2}+\right. & \left.\varepsilon_{\perp} q_{\perp}^{2}\right) V_{q}= \\
& =4 \pi\left\{\left(\mathrm{e}_{1}+\mathrm{e}_{3}\right) q_{\perp}^{2}+\mathrm{e}_{2} q_{z}^{2}\right\} \gamma_{q} .
\end{aligned}
$$

La correction à l'énergie libre $\left(\right.$ par $\left.\mathrm{cm}^{3}\right)$ est :

$$
\Delta F=\frac{\mathbf{E} \hat{\varepsilon} \mathbf{E}}{8 \pi}
$$


où $\mathbf{E}=-\nabla V$ est le champ électrique. Pour une composante de Fourier, ceci donne :

$$
\Delta F_{q}^{\mathrm{j}}=\frac{2 \pi\left\{\left(\mathrm{e}_{1}^{\mathrm{q}}+\mathrm{e}_{3}^{\mathrm{T}}\right) q_{\perp}^{2}+\mathrm{e}_{2} q_{z}^{2}\right\}^{2}}{\varepsilon_{\|} q_{z}^{2}+\varepsilon_{\perp} q_{\perp}^{2}}\left|\gamma_{q}\right|^{2} .
$$

Dans la forme non perturbée (éq. I.6) on avait

$$
\frac{1}{2} \bar{B} q_{z}^{2}\left|u_{q}\right|^{2} \equiv \frac{1}{2} \bar{B}\left|\gamma_{q}\right|^{2} \text {. }
$$

En ordre de grandeur, $\Delta F_{q}$ est plus petit que ce terme par un facteur $q^{2} d^{2} \ll 1$. Donc les effets piézoélectriques sont négligeables [12].

\section{Bibliographie}

[1] Chistyakov (G.), Soviet Physics Uspekhi, 1967, 9, 551. Le cas des phases smectiques de lipides doit être disjoint, ces matériaux ayant fait l'objet d'études intensives : voir la revue par V. LuzzATI à cette conférence.

[2] Oseen (C. W.), Trans, Faraday Soc, , 1933, $29,883$.

[3] Frank (F. C.), Disc. Faraday Soc., 1958, 25, 1.

[4] Landau (L. D.), Lifshitz (I. M.), Statistical Physics, (Pergamon Press, Londres, 1958), p. 411.

[5] De Gennes (P. G.). Proceedings of the 2nd Kent Conference on liquid Crystals (à paraître dans Molecular Crystals).

[6] De Gennes (P. G.), J. Chem. Phys., 1968, 48, 2257.

[7] Groupe D'Orsay, J. Chem. Phys., 1969, 51, 816.

[8] De Gennes (P. G.), C. R. Acad. Sci. Paris, 1968, 266, 15.

[9] Chatelain (P.), Acta crystallographica, 1948, 1, 315.

[10] Pour le mode (( péristaltique ) des films de savon, qui est un peu comparable à l'ondulation décrite ici, le régime est probablement isotherme : voir : $D E$ Gennes (P. G.), C.R. Acad. Sci. Paris, 1969, 268 B, 1207.

[11] MeYer (R. B.), Phys. Rev. Lett., 1969, 22, 918.

[12] La situation est tout à fait différente dans un nématique, où l'intensité de la lumière diffusée peut être fortement affectée par les effets piézoélectriques. Groupe D'Orsay, à paraître.

\title{
ÉTUDE, PAR DIFFUSION QUASI-ÉLASTIQUE DE LA LUMIÈRE, DE L'AMORTISSEMENT DES FLUCTUATIONS THERMIQUES D'ORIENTATION MOLÉCULAIRE DANS UN CRISTAL NÉMATIQUE
}

\author{
Groupe d'étude des cristaux liquides d'Orsay
}

\begin{abstract}
Résumé. - Nous reprenons les expériences de Chatelain [1] de diffusion Rayleigh par un monocristal nématique, en observant non plus l'intensité, mais le spectre de la lumière diffusée, à l'aide d'un spectromètre laser à battements optiques. Nous isolons les deux modes de relaxation pure, basse fréquence, récemment prévus par le groupe d'étude des cristaux liquides d'Orsay [2], superposition de " flexion 》, ( torsion 》 et " divergence 》 de l'axe optique. Nos premiers résultats [3] sur la dépendance angulaire des spectres obtenus nous permettent de déterminer trois des six coefficients introduits par Leslie [4] pour décrire la viscosité d'un nématique.
\end{abstract}

Abstract. - The Rayleigh scattering in nematic liquid crystals was first studied by Chatelain [1] who investigated the angular dependence of the scattered light intensity. Resuming this experiment, we have now observed, not the intensity, but the power spectrum of the scattered light, using an optical beat laser spectrometer. Intensity fluctuations are caused by thermal distortions of the nematic monocrystal, superposition of " bending », " twist, ) and ( splay » of the optical axis. We identify the two low frequency purely dissipative modes recently predicted [2]. A preliminary study [3] of the angular dependence of the width of these modes allows a determination of three among the six viscosity coefficients introduced by Leslie [4].

\section{Références}

[1] Chatelain (P.), Acta Cryst., 1948, 1, 315.

[2] Groupe D'Étude des CRISTAuX LIQUides D'Orsay, $J$. Chem. Phys., 1969, 51, 816.

[3] ORSAY LIQUID CRYSTAL GROUP, Phys. Rev. Letters, 1969, 22, 227.

[4] Leslie (F. M.), Quant. Journ. Mech. \& Applied Math., $1966,19,337$. 